\title{
UNILATERAL LIMB LENGTHENING ASSOCIATED WITH HAEMATOGENOUS OSTEITIS IN CHILDHOOD
}

\author{
BY \\ WALLACE M. DENNISON \\ From a Surgical Unit, Royal Hospital for Sick Children, Glasgow
}

(RECEIVED FOR PUBLICATION MAY 2, 1951)

Increase in girth of a long bone following pyogenic inflammation is well known, but there is little recent literature describing increase in length following prolonged bone infection. In a recent series of 100 cases of acute haematogenous osteitis treated with penicillin the disease affected a long bone on 84 occasions. Measurable increase in length of the affected limb occurred in 20 cases. During the same five-year period increase in length of the lower limb was also noted in conditions other than pyogenic bone infection.

The effects of alteration in circulation on bone growth have interested many observers. In the sixteenth century Ambroise Paré noted that stasis hyperaemia stimulated bone growth, and he employed hyperaemia to stimulate callus formation in fractures. Three centuries later Thomas (1886) rediscovered the value of venous stasis in fractures with retarded callus formation. Stanley (1849), Paget (1853) and Bergmann (1868) showed that any long-continued inflammatory process would be followed by increase in length and girth of the bones. Bier (1905) referred to the effects of stasis hyperaemia on bone growth and his book furnishes an excellent historical review of this subject. In recent works on bone disease and fractures there is little reference to increase in length of a long bone produced by circulatory derangement.

Although limb lengthening is most commonly a sequel to haematogenous osteitis, increase in length of the lower limb was noted in the following conditions seen in one unit of the Royal Hospital for Sick Children, Glasgow, during the past five years : (1) $2 \mathrm{~cm}$. lengthening after simple transverse fracture of the femur; (2) $3 \mathrm{~cm}$. lengthening associated with a diffuse angiomatous condition of one leg (congenital arteriovenous fistulae); (3) $2 \mathrm{~cm}$. lengthening after recurrent haemarthrosis of one knee in a child suffering from haemophilia; (4) $2.5 \mathrm{~cm}$. lengthening after severe lacerations of the thigh and calf; (5) $2.5 \mathrm{~cm}$. lengthening in a child suffering from synovial tuberculosis of the hip; and (6) $3 \mathrm{~cm}$. lengthening associated with congenital neurofibromatosis (biopsy revealed plexiform dilatation of veins). In all these conditions there is a common factor of increased blood supply. Leriche and Policard (1926) have suggested an indirect vascular effect on bone growth by the sympathetic nervous system. We have found an increase in length of one lower limb of at least $1 \mathrm{~cm}$. following unilateral lumbar ganglionectomy performed for various conditions.

Before the introduction of penicillin in the treatment of osteitis gross limb lengthening was common and was due to the continuing hyperaemia caused by the presence of an infected sequestrum. Although sequestrum formation was much less common in the penicillin-treated cases, it still occurred (Dennison, 1948) and Figs. 1 and 2 show examples of the resultant increase in length of the affected limb. Where only one of two paired bones was diseased, the increase in length affected both bones equally (Figs. 3 and 4). The hyperaemia presumably affects the soft tissues and increases the blood supply throughout the segment. Irregular growth occurred when there was damage to one part of the epiphysis (Figs. 9 and 10). The increase in length varied from 1 to $3 \mathrm{~cm}$. and the lengthening usually became obvious eight to 12 months after the onset of the disease. The patients were all under 13 years of age at the time of onset of the bone infection.

Sterile sequestra are usually absorbed (Bancroft, 1921) but an infected sequestrum unless extruded must be removed surgically. In 25 successive sequestrectomies performed during the past five years, coagulase positive staphylococci have been grown from either the sequestrum or the surrounding granulation tissue in 24 cases. Advantage of this knowledge was taken in one case in the penicillintreated series. Pathological fracture of the upper and lower ends of the femur complicating severe bipolar osteitis was followed by $1 \mathrm{~cm}$. shortening of the affected leg (Fig. 5). Radiography revealed a cavity with sequestrum formation, and at operation 

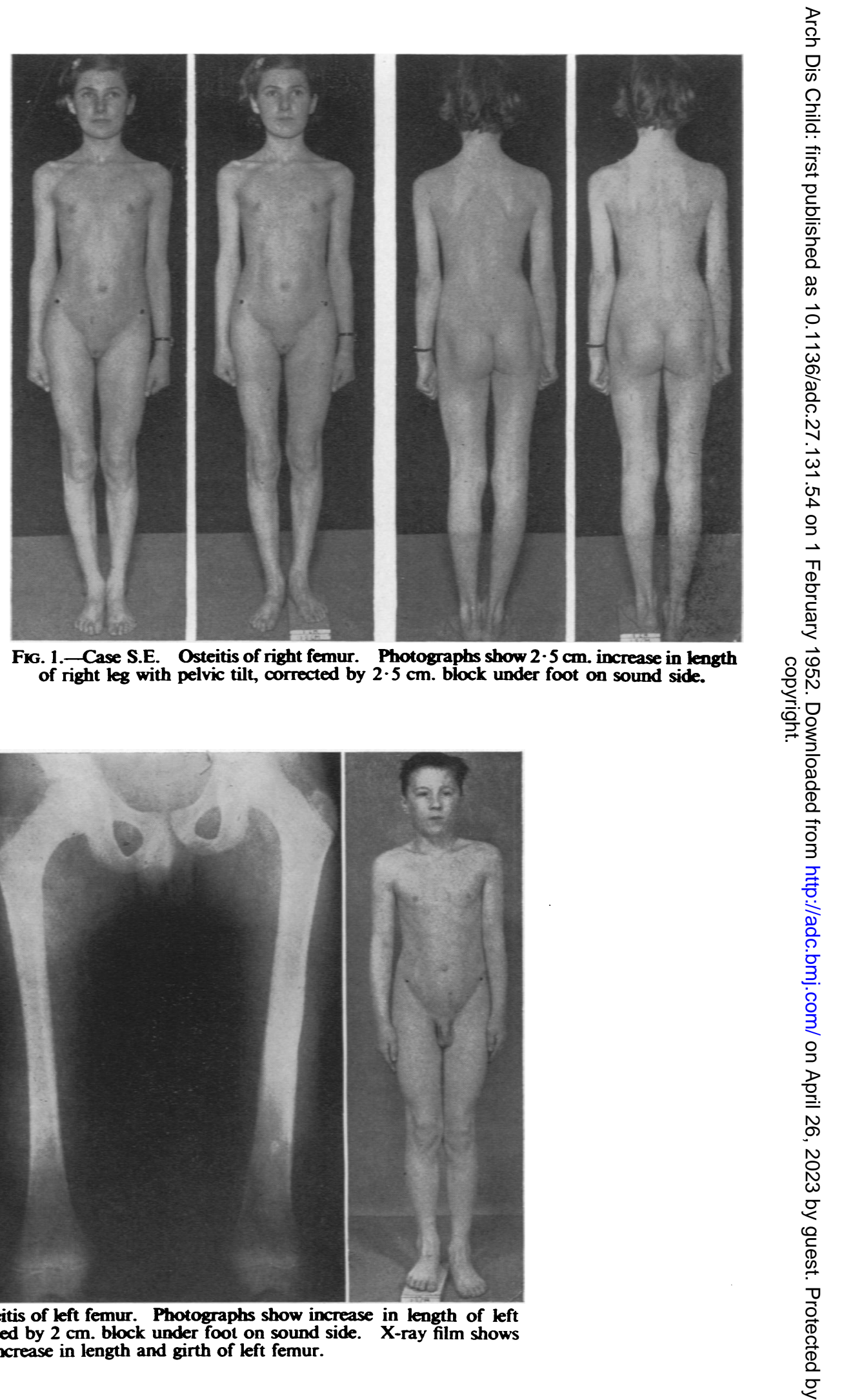

Fig. 2.-Case J.H. Osteitis of left femur. Photographs show increase in length of left leg and pelvic tilt, corrected by $2 \mathrm{~cm}$. block under foot on sound side. X-ray film shows increase in length and girth of left femur.

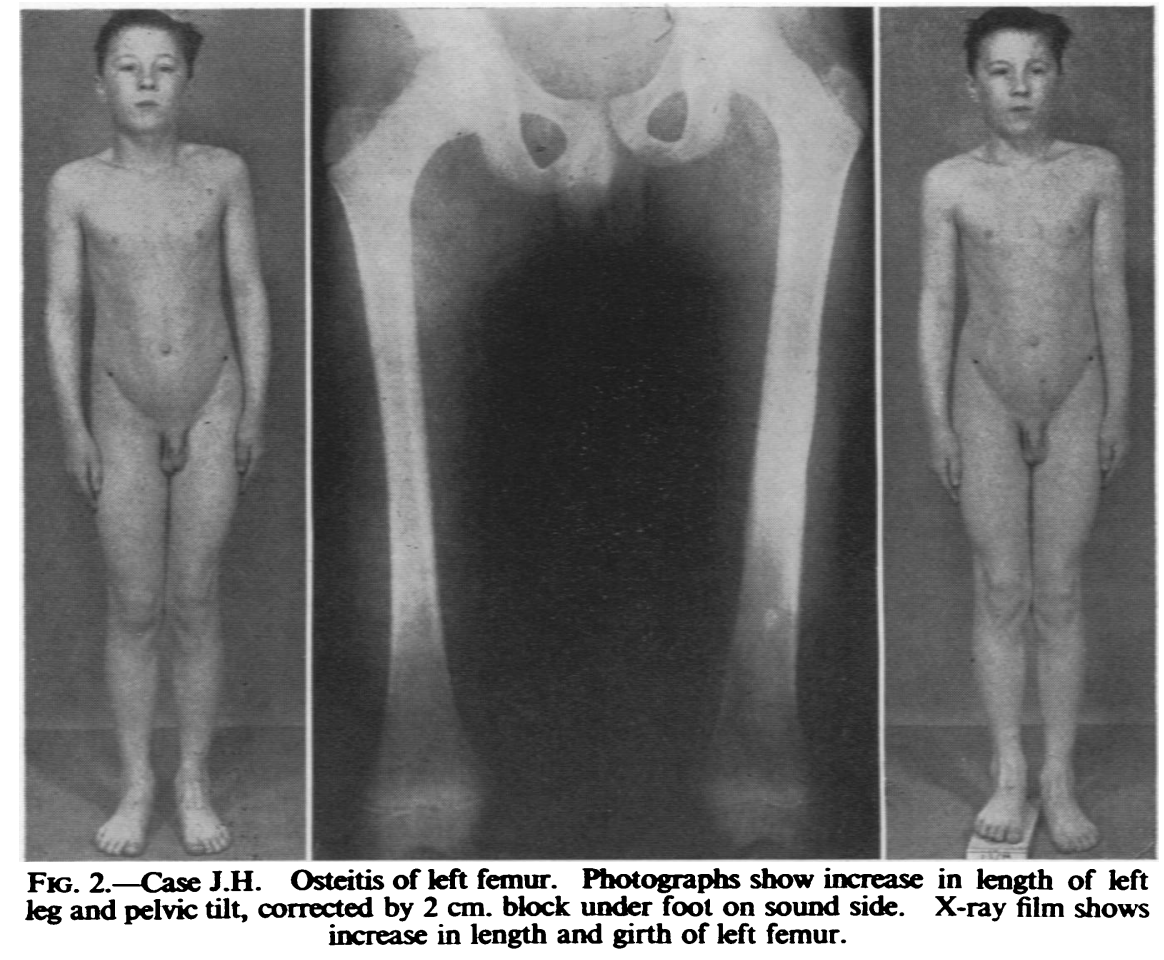

G. 1. Case S.E. Osteitis of right femur. Photographs show $2.5 \mathrm{~cm}$. increase in length of right leg with pelvic tilt, corrected by $2.5 \mathrm{~cm}$. block under foot on sound side. 


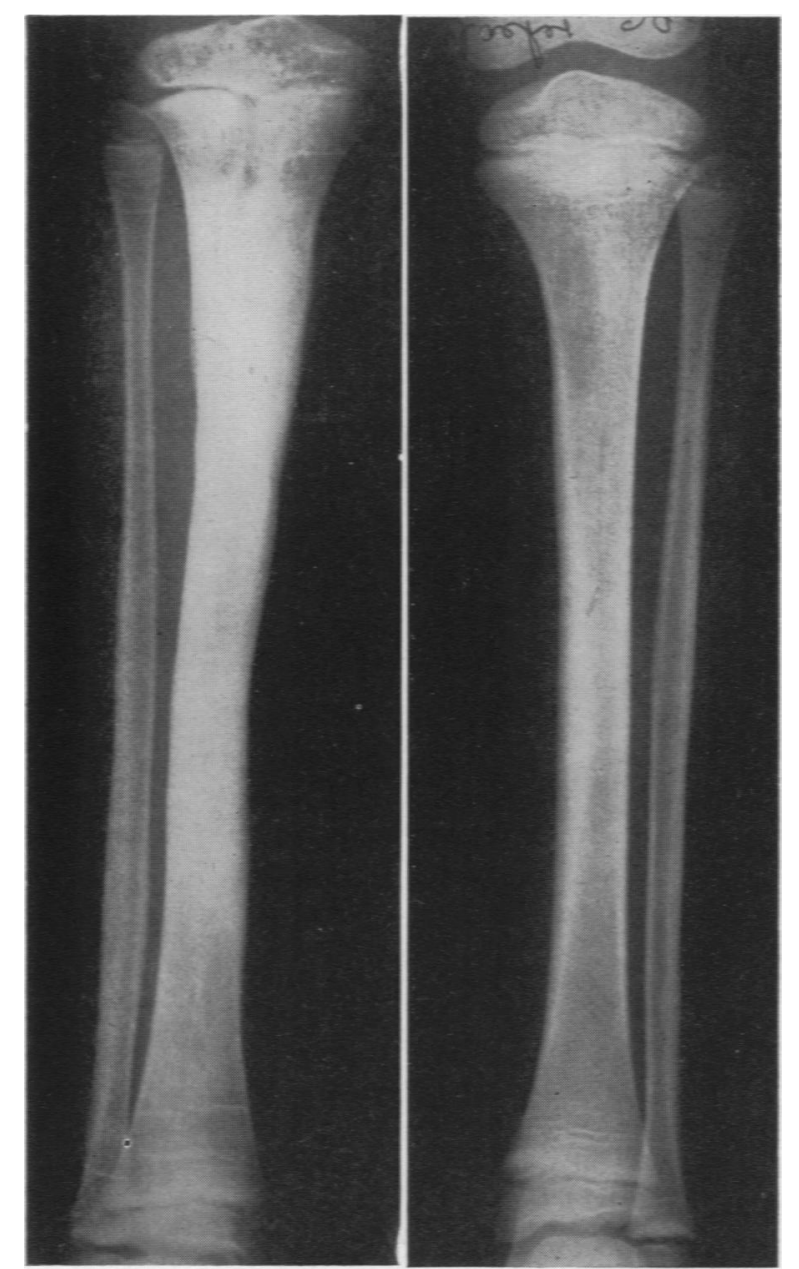

FIG. 3.

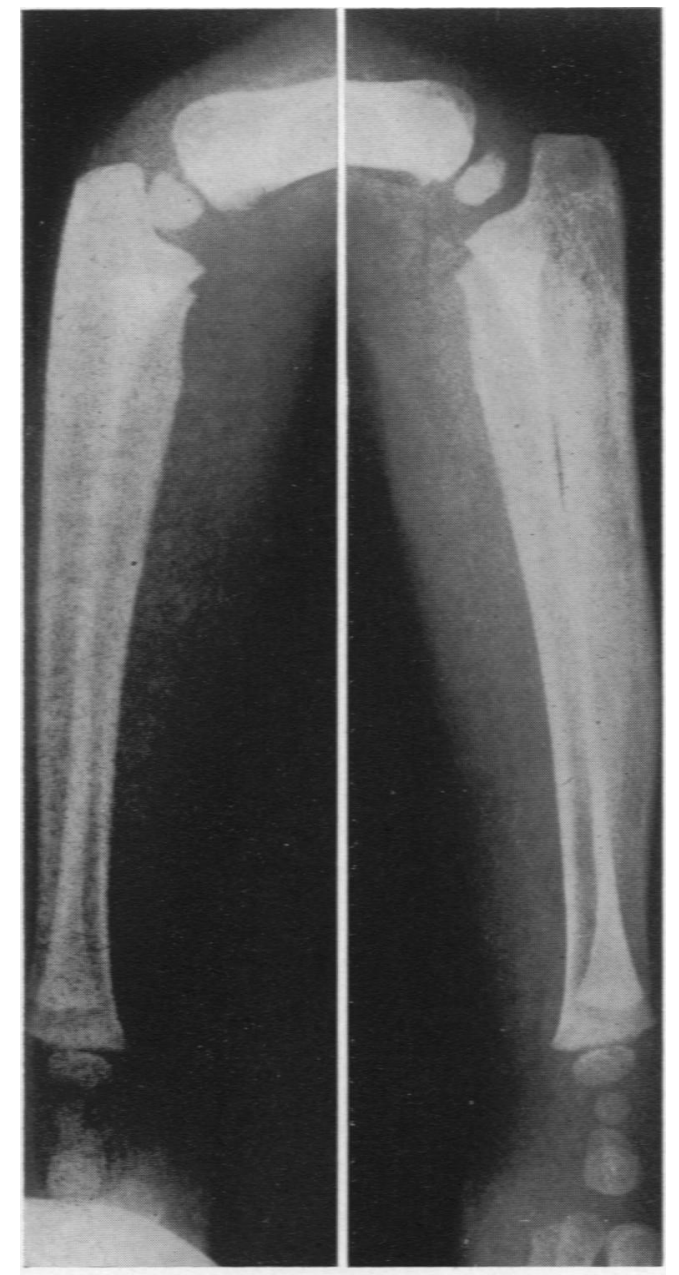

FIG. 4.

Fig. 3--Case J.G. Osteitis of right tibia. Radiograph of both tibiae on one film, 18 months after onset of acute $N$ osteitis, showing sclerosis of right tibia and increase in length of both right tibia and fibula.

$N$
N
ర్

Fig. 4.-Case M. McH. Osteitis of ulna. Radiograph $\Theta$ film of both forearms, showing increase in girth of right $\frac{}{D}$ ulna and increase in length of right ulna and radius, two $\stackrel{\mathscr{S}}{+}$ years after onset of acute osteitis. 


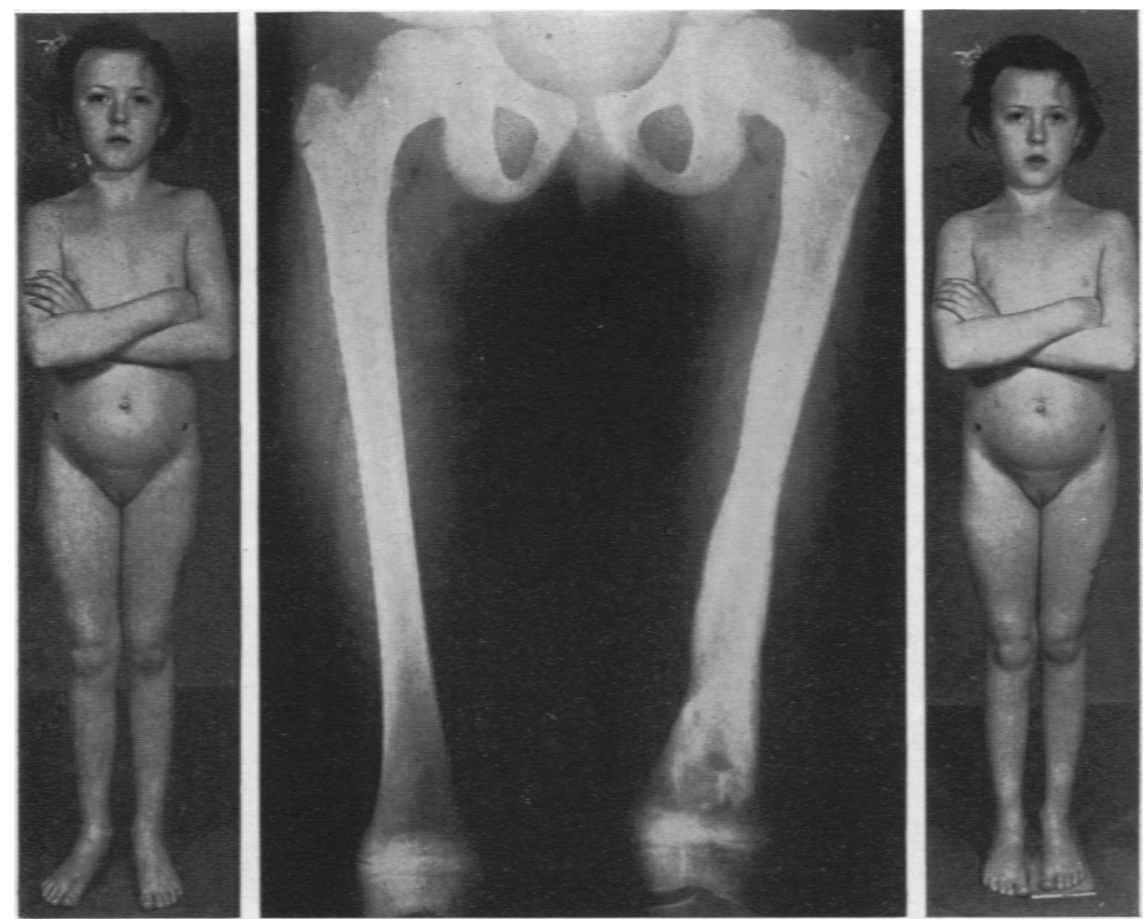

FKG. 5.-Case M.H. Photographs show shortening of left leg and slight pelvic tilt corrected by $1 \mathrm{~cm}$. block under left foot. X-ray film shows coxa vara following pathological fracture of the neck of the left femur, sclerosis of the shaft and cavitation in lower quarter.

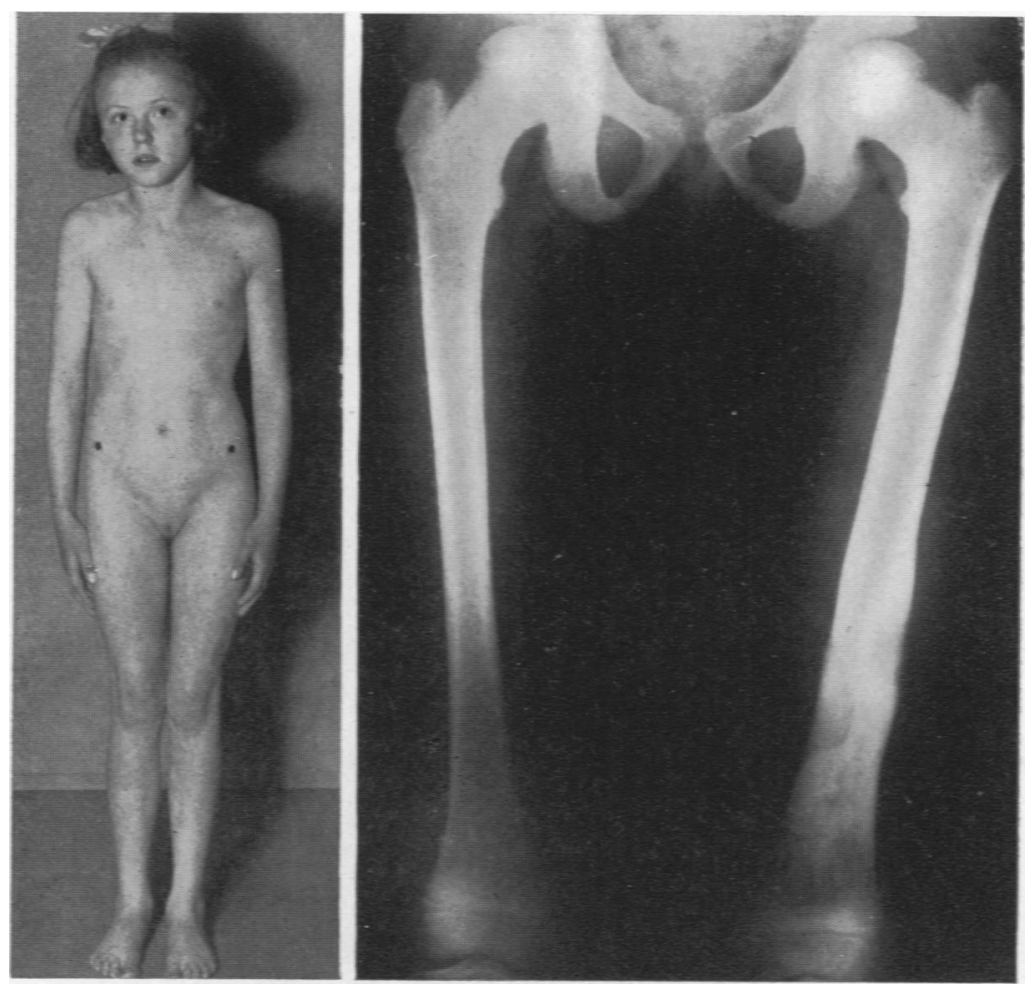

Fig. 6. - Case M.H. (eight months later). Legs of equal length in photograph and $x$-ray film. 
FIG. 7.-Case J.P. Osteitis of right femur. Four years after onset. Photographs show scoliosis and genu valgum partially corrected by $2 \mathrm{~cm}$. block under foot on normal side. $\mathrm{X}$-ray film shows sclerosis and increase in length and girth of right femur.
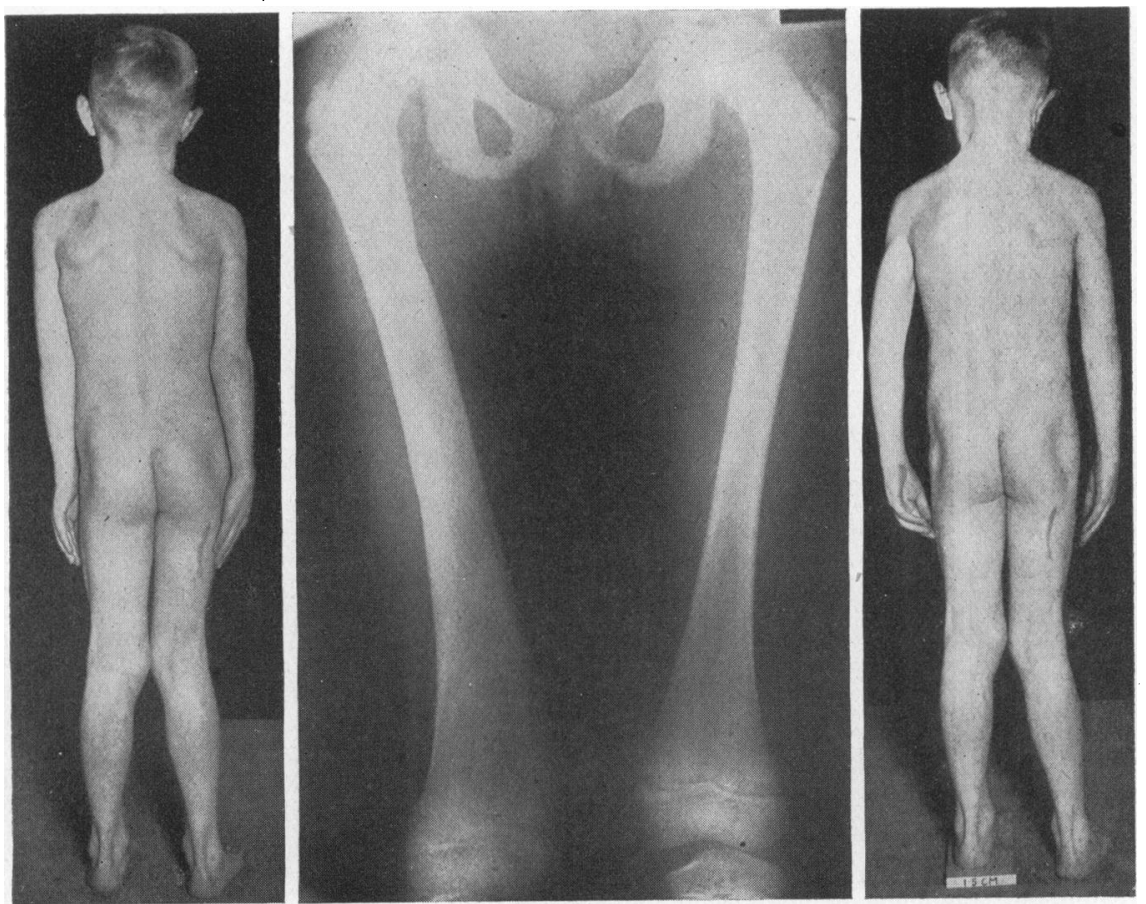

FIG. 8.-Case L. McI.

Osteitis of right femur. Photographs show genu valgum following overgrowth of right femur. X-ray film shows sclerosis and increase in girth and length of right femur. 
on February 12, 1949, three small infected sequestra were removed. A fourth sequestrum was deliberately left in situ to promote bone growth. On October 4 the remaining sequestrum was removed and Fig. 5 (October 16) shows both legs of equal length.

The growth stimulus over-activated by the increased blood supply gradually passes off and in the actively growing child a moderate increase in length (up to $2 \mathrm{~cm}$.) is rectified within five years. In a series of cases of osteitis treated before the introduction of chemotherapy and followed up until adult life (10-15 years later) an increase in length of $3 \mathrm{~cm}$. noted between 1936 and 1940 was no longer present in 1950 . Presumably growth ceases earlier in the overgrown limb.

It must be remembered that the growing child can compensate for a long lower limb by such postural defects as scoliosis (Fig. 7) and genu valgum (Fig. 8). Constant watch must be kept on the patient as such deformities develop insidiously over a period of years if suitable raising is not applied to the footwear of the normal leg.

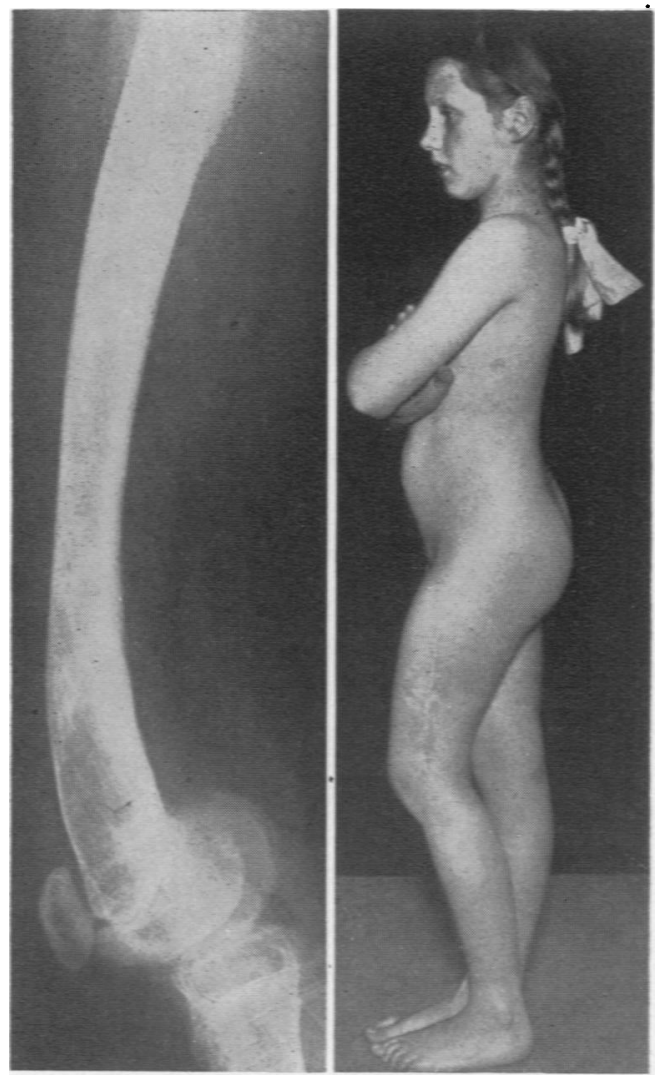

FIG. 9.-Case B.M. Osteitis of left femur. Flexion deformity following damage to lower femoral epiphysis.

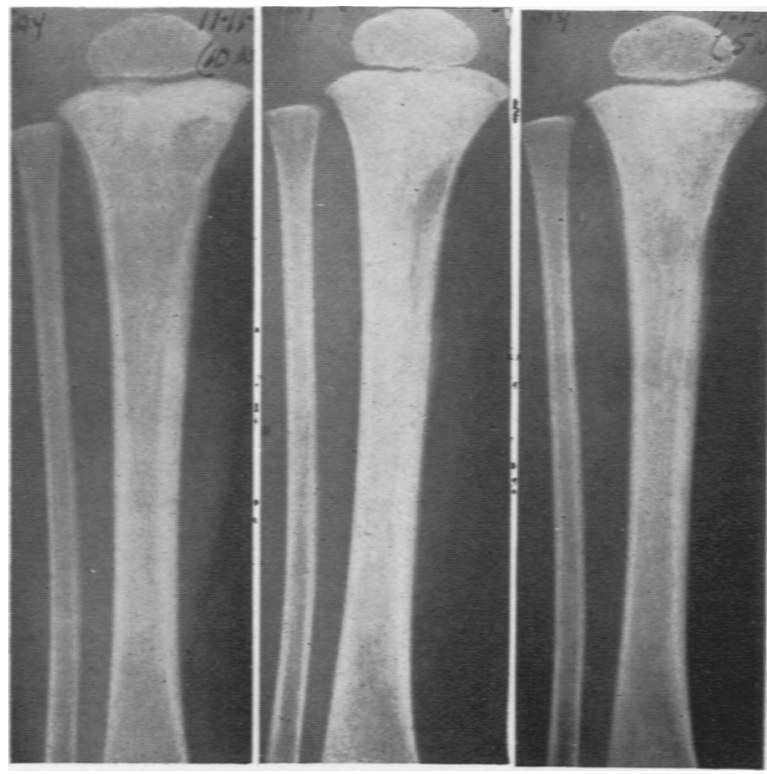

FIG. 10.-Case K.M. Osteitis of right tibia (b) two weeks after onset: (c) five weeks after onset; (a) seven months after onset, overgrowth of medial tibial condyle causing genu valgum.

\section{Summary}

In 100 cases of acute haematogenous osteitis the disease affected a long bone on 84 occasions. Measurable increase in length of the affected limb occurred in 20 cases.

Limb lengthening occurs in other conditions in which there is an increased blood supply to the part. Examples of such conditions are mentioned.

Unless appropriate action is taken secondary postural deformities may follow increase in length of one lower limb.

The cases were all studied in Mr. White's surgical wards and I am grateful to him for his helpful advice and criticism. I wish to thank Mr. J. L. A. Evatt, A.R.P.S., medical photographer, Royal Hospital for Sick Children, Glasgow, for the photographs and reproductions of $x$-ray films.

\section{REFERENCES}

Bancroft, F. W. (1921). Ann. Surg., 73, 681.

Bergmann, E. (1868). St Peters. med. Z., 14, 65

Bier, K. G. A. (1905). Hyperemia as a Therapeutic Agent.' (Trans.), edited by G. M. Blech, Chicago.

Dennison, W. M. (1948). J. Bone Jt Surg.. 30B, 110. Leriche, R. and Policard, A. (1926). Surg. Gynec. Obstet., 43, 308.

Paget, J. (1853). 'Lectures on Surgical Pathology.' London.

Paré, A. 'Oeuvres complètes d'Ambroise Paré,' 1840-41. Paris.

Stanley, E. (1849). A Treatise on Diseases of the Bones.' London.

Thomas, H. O. (1886). Contributions to Surgery and Medicine, Part 6: The Principles of the Treatment of Fractures and Dislocations.' London. 